\title{
Gas fired boilers: Perspective for near future fuel composition and impact on burner design process
}

\author{
Fabio Schiro ${ }^{1, *}$, Anna Stoppato $^{1}$, and Alberto Benato ${ }^{1}$ \\ ${ }^{1}$ University of Padova, Department of Industrial Engineering, Via Venezia n.1, Padova, Italy
}

\begin{abstract}
The advancements on gas boiler technology run in parallel with the growth of renewable energy production. The renewable production will impact on the fuel gas quality, since the gas grid will face an increasing injection of alternative fuels (biogas, biomethane, hydrogen). Biogas allows producing energy with a lower $\mathrm{CO}_{2}$ impact; hydrogen production by electrolysis can mitigate the issues related to the mismatch between energy production by renewable and energy request. These technologies will contribute to achieve the renewable production targets, but the impact on whole fuel gas production-to-consumption chain must be evaluated. In the first part of this study, the Authors present the future scenario of the grid gas composition and the implications on gas fed appliances. Given that the widely used premixed burners are currently designed mainly by trial and error, a broader fuel gas quality range means an additional hitch on this design process. A better understanding and structuring of this process is helpful for future appliance-oriented developments. The Authors present an experimental activity on a premixed condensing boiler setup. A test protocol highlighting the burners' flexibility in terms of mixture composition is adopted and the system fuel flexibility is characterized around multiple reference conditions.
\end{abstract}

\section{Introduction}

Heating is a fundamental necessity especially in countries with cold climates, and gas fired boilers are currently satisfying large part of this heat demand in the domestic sector. Gaseous fuels are forecasted to be widely used to feed gas boilers in the next years [1]. Among them, premixed boilers allow to obtain higher energy efficiency and lower pollutant emission with respect to non-premixed boilers [2]. For this reason, premixed boilers are becoming mandatory in the EU except for special circumstances [3]. Significant efforts have been done in order to develop and industrially apply the technology of these devices. At the same time, a significant commitment was placed on increasing renewable energy production. The renewables production will have a direct impact also on the quality of the fuel gas feeding heating appliances in the near future, since the gas grid will face an

\footnotetext{
*Corresponding author: fabio.schiro@dii.unipd.it
} 
increasing injection of alternative fuels (mainly biogas, biomethane, hydrogen) [4-7]. In particular, hydrogen production from electrolysis can mitigate, on the one hand, the issues related to the fluctuations in renewable energy production (particularly massive in the wind farms) and, on the other hand, can help to cover the mismatch between energy offer and request. This path will reduce the overall $\mathrm{CO}_{2}$ emission and contribute to achieve the renewable production targets, but the impact on every element of the production-toconsumption chain need to be evaluated. In the first part of this study the Authors discuss the future scenario of the grid fuel gas compositions. A qualitative evaluation of the implications on premixed burners design will be conducted basing on existing studies. Premixed boilers will be the leading technology for gas heating in the next future and burner design is currently driven by trial and error methodology [8-9]. A broader fuel gas quality range means an additional hitch on the burner design process: a better understanding and structuring of the today's burner design process would be helpful in the future appliance-oriented research and developments. In the second part of the paper, the Authors present an experimental performance assessment of cylindrical premixed burners coupled to a very common commercial premixed boiler setup. A test protocol highlighting the burners' flexibility in terms of mixture composition is adopted and the system fuel flexibility is characterized around multiple reference conditions.

\section{Perspective for future grid fuel composition}

\subsection{General context}

Gaseous fuels composition is expected to vary in the next years, and the existing gas quality standards need to evolve in parallel. Guiding factors for this process are the security of energy supply and the reduction of overall $\mathrm{CO}_{2}$ emission.

\subsection{Hydrogen}

The recent developments in renewables, especially in wind power farms, make the electricity storage issue more and more important for the future energy system efficiency. Among the most trusted and consolidated storage systems are the pumped-hydro energy storage and the CAES, whose potential is anyway limited due to geographical and technical reasons. An option being intensively studied is the storage of the overabundant electricity into hydrogen, produced by electrolysis, and the subsequent injection through the natural gas network. This technology is often reported as P2G (Power To Gas). It allows to use an already existing huge and geographically branched energy storage system, and potentially avoids to build new electricity transmission lines. Hydrogen volume percentages up to $30 \%$ have been taken into consideration according to certain studies [10]. In fact, today, it is necessary to improve the energy and economic efficiency of renewable production, since not all the electricity grids are able to transport the energy produced during the peaks of wind and sun, with the consequent need of forcing the production shut down to avoid electricity grid overloading. Obviously, the hydrogen injected into the gas network could also contribute to produce heat and power in distributed or centralized plants. [4]

\subsection{Biogas}

The European Union encourages the use of biomass as renewable energy source, in the perspective of greenhouse gases reduction. Biogas is a fuel produced by anaerobic digestion 
of organic matters, such as animal slurries and energy crops, and it is mainly composed by methane and carbon dioxide. The composition depends on numerous factors such as the process design and the nature of the digested substrate: table 1 reports data from a literature datasheet [11] on fuel properties. Often, biogas fuel is used to produce heat and/or power in boilers or cogeneration units near the biogas production plant. However, an alternative is the injection of biogas into the natural gas grid [5-7] after a treatment process, allowing to use the grid itself for long distance transport and to balance fuel demand and supply by storage.

Table 1. Relevant compositional data of biogas and natural gas [11]

\begin{tabular}{|c|c|c|}
\hline & Biogas & Natural gas \\
\hline & & $85-92$ \\
\hline Methane [Vol\%] & $60-70$ & $0-9$ \\
\hline Heavy hydrocarbons [Vol\%] & 0 & $0.3-1.0$ \\
\hline Nitrogen [Vol\%] & 0.2 & $0.2-1.5$ \\
\hline Carbon dioxide [Vol\%] & $30-40$ & $1.1-5.9$ \\
\hline Hydrogen sulphide [ppm] & $0-4000$ & \\
\hline
\end{tabular}

\subsection{Security of supply and strategic aspects}

The security of fuel gas supply is a fundamental need especially in Europe after the reduction of fuel reserves and the growth of domestic fuel demand (which is recovering from the economic crisis and forecasted to further increase [1]). In this context, the fuel gas demand can be satisfied by the already mentioned renewable gas production (biogas, biomethane, hydrogen) or by increasing the dependency from foreign energy imports (LNG or pipeline imports). This situation involves both environmental and strategic evaluations, and will add variables to the fuel gas composition, that will be made up by a combination among indigenous gases, renewable gases, imported pipeline or LNG gases [12].

\section{Impact on gas devices and on burner design process}

Gas boilers and other gas fed appliances are tested, basing on standards, with a reference gas and extreme limit gases [13] (e.g. incomplete combustion and soot limit gas, light back limit gas, flame lift limit gas, overheating limit gas). These limit gases are designed to guarantee a safe operation under variations in gas compositions within the appliance lifetime. The limit gases, anyway, do not represent the allowed distribution gases, and the standard does not define which distributed gas composition and Wobbe index range is covered by the limit gases testing. Distribution gas ranges are usually defined as Wobbe index range, but compositional effects (non-methane hydrocarbons, hydrogen, $\mathrm{CO}_{2}, \ldots$ ) play also a key role on safe operation. Regarding the distribution range, each European country made decisions based on own practice and experience [14]. Due to the complex scenario, any variation on the gas quality standards must be carefully studied and evaluated. Several studies $[12,15,16]$ are focused on understanding the impact of gas quality variations on gas appliances: pollutant emissions (CO, NOx) are related to the fuel quality and potential safety risks have been identified. The specific theme of hydrogen addition has also been explored in literature with reference to domestic appliances $[10,17,18]$. Hydrogen significantly impacts on the flame dynamics: flame lift, noisiness and combustion control issues were detected in laboratory testing of existing devices. The varied combustion chemistry and consequent different flame speed influenced also pollutant emissions, burner 
temperature and consequent burner lifetime. Therefore, basing on the above-mentioned works, it is possible to underline that each application has its own characteristic response to the different gas quality specification. The possible future modifications of device design in order to increase the tolerance on gas composition and heating value will require an even more tailor-made approach than that current applied [17]. Active combustion control systems could mitigate the required modifications to application, but these systems were also found to be not completely mature to deal with all fuel components such as hydrogen $[10,18]$. In conclusion, it is highly probable that to widen the gas specification range an increased fuel flexibility would be required to the gas burner itself.

\section{Experimental activities on premixed boiler fuel flexibility}

\subsection{Introduction}

Based on the scenario presented in Section 2 and 3, a consistent research and optimization activity on gas fed appliances is necessary to grant safe operation and full functionality under future boundary conditions. The Authors focused on domestic premixed boilers, which will be widely used for emission and efficiency reasons and whose burner design is particularly complex. The burner is a key component for these devices, because it significantly influences the combustion performance; however trial and error approach still drives the premixed burner design [8-9]. The combustion dynamics governing these burners are not fully understood. Therefore, the design process is founded on manufacturer's knowhow and on empirical observations. Additional difficulties are added to the burner development phase by budget, time and production cost factors [19]. These difficulties would be even worsened by the forecasted wider grid gas quality range, discussed in detail in the previous sections. In fact, if the grid fuel gas composition will be more variable in the future, the premixed burners need to correctly operate under the consequent more variable air/gas ratio without problems. In this context, it is useful to generate knowledge on the premixed burners behaviour under variable fuel quality, with the aim of assisting future appliance-oriented burner design processes. The Authors focused on a domestic premixed boiler system and implemented a test protocol, with the aim to overcome the mere pollutant emission testing with nominal gas and with limit gases. An in-depht analysis has been conducted with the aim of gathering more information on combustion behaviour at gradually varying air/gas mixture composition. Emission and flame stability characteristics have been identified.

\subsection{Experimental setup}

The test rig adopted in the experiments replicates the fundamental structure of a domestic premixed boiler: pneumatic gas valve, modulating blower, Venturi effect air/gas mixer, steel coil heat exchanger, cylindrical burner. A $25 \mathrm{~kW}$ nominal thermal capacity has been chosen because it is very common among domestic boilers [20]. A front view of the test rig used is visible in Figure 1. 


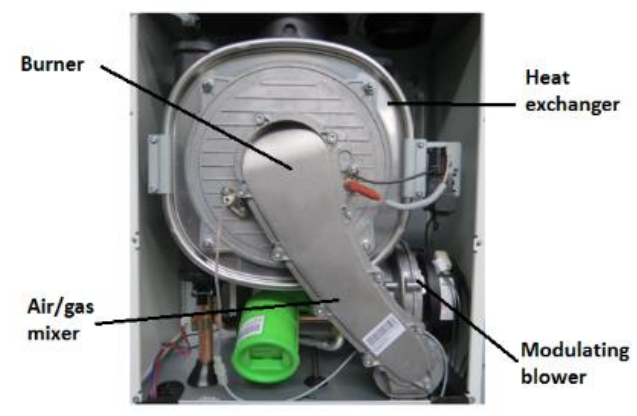

Fig. 1. Front view of the test rig used for the experiments

The emission values reported in this paper are normalized to the air free reference condition. Gaseous emissions are measured by infrared-based analysers (SIEMENS Ultramat 6 for $\mathrm{CO}$ and $\mathrm{CO}_{2}$, repeatability $<1 \%$ of respective measuring range, linearity deviation $<0.5 \%$ of full-scale values) and chemiluminescence based analysers (Eco Physics CLD 82 Sh for NOx, repeatability $<0.5 \%$ of reference point, lack-of-fit $<0.8 \%$ of full-scale value). Gas flows are measured by thermo-mass meters (Brooks SLAMF, repeatability < $0.2 \%$ of reference point, flow accuracy $\pm 0.9 \%$ of full scale value). For the aim of this study an industrially available premixed steel burner (see figure 2) equipping an on-the-market 25 $\mathrm{kW}$ domestic boiler was chosen as reference combustor. Such a burner is designed to fulfil several target requirements, including overall dimensions, pressure drop value, thermoacoustic behaviour, flashback resistance, and lifetime.

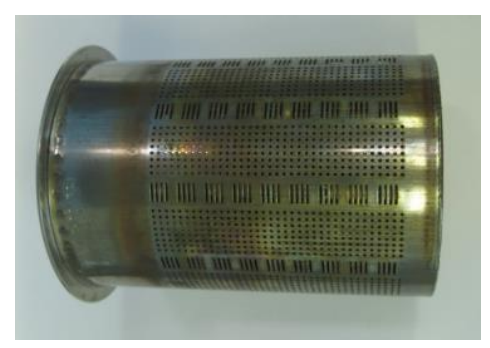

Fig. 2. The reference steel premix burner

\subsection{Test protocol}

In order to evaluate the response of the system to a fuel gas quality variation and to characterize its flexibility, a test protocol has been developed. Three reference thermal load conditions (maximum load, average load, minimum load) have been selected in a thermal load modulation range of $10: 1$, and the air/fuel ratio is gradually varied in correspondence to each of these conditions. The $\mathrm{CO}$ emission is taken as marker of flame stability and combustion hygiene while the $\mathrm{CO}_{2}$ emission is directly linked to the fuel/air ratio. Operatively, the boiler fan speed is fixed for each thermal load reference condition to elaborate a fixed air flow while the gas valve setting is gradually varied to regulate the fuel flow. In this way, the response of the boiler to a variable fuel/air ratio mixture is simulated. Experimental results are acquired when the system is in steady-state condition. The fuel gas used is pure methane, but the same methodology can be applied also with other fuel components. The nominal reference conditions are listed in Table 2. The nominal air/gas 
ratio has been tuned in order to achieve a $9 \% \mathrm{CO}_{2}$ level in the exhaust: this is a typical value for this kind of boilers.

Table 2. Reference testing conditions

\begin{tabular}{|c|c|c|c|}
\hline Reference condition & Thermal load [kW] & $\mathrm{CO}_{2}$ setting [\%] & Fan speed [rpm] \\
\hline & & & \\
\hline Maximum load & 25.0 & 9.0 & 5100 \\
\hline Average load & 12.7 & 9.0 & 3000 \\
\hline Minimum load & 2.5 & 9.0 & 1100 \\
\hline
\end{tabular}

\subsection{Experimental results}

The $\mathrm{CO}$ values versus the $\mathrm{CO}_{2}$ levels for each condition are depicted in Figure 3 .

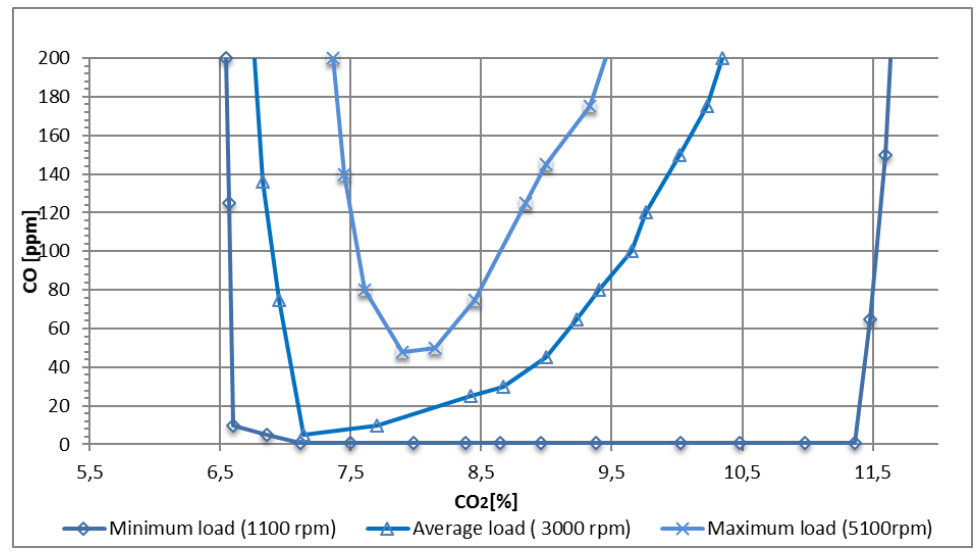

Fig. 3. $\mathrm{CO}$ versus $\mathrm{CO}_{2}$ curves at reference fan speeds

The $\mathrm{CO}$ versus $\mathrm{CO}_{2}$ assumes a macroscopic common shape for all reference conditions: we will refer to this shape in the following with the name "U curve". This curve presents two quickly ascending branches and a region in which the $\mathrm{CO}$ emission is low and more or less constant. These results can be referred to some basic premixed combustion mechanisms. The $\mathrm{CO}$ increases for low $\mathrm{CO}_{2}$ values (lean mixtures) because at low gas/air ratio the flame is long, prone to stretching and lifting. Thus, it is affected by edge effects and potential quenching through the heat exchanger walls. The $\mathrm{CO}$ increases for high $\mathrm{CO}_{2}$ values (rich mixtures), on the other side, because increasing gas/air ratio the oxidizer concentration decreases, thus incomplete combustion is favoured. The $\mathrm{CO}$ increment for rich mixture conditions is smoother than for lean ones: this is linked to the above-mentioned phenomena, since flame lift condition can be sudden in response to a small ratio variation and quenching through the walls could play a significant role. The results confirm that the gas/air ratio has a fundamental impact on the combustion quality of a premixed boiler. Thus fuel gas composition variations shifting this ratio can significantly increase the CO production. In addition, the $U$ curves get narrowed passing from minimum thermal load through maximum load, suggesting that the decrease of combustion products residence time and the increase of flame length generally disadvantage combustion completion. The fuel flexibility result to be wider al lower thermal load: this suggests that appliance developers should take care about fuel flexibility along whole thermal load modulation range, since 
emission level and behaviour can significantly differ. The proposed U-curve tracing is a fundamental first step to characterize the system fuel flexibility: comparing the mixture ratio tolerance required for the appliance and the obtained $U$ curve an evaluation on the burner flexibility can be drawn. This method allows also to detect the preferable air/gas ratio with the aim to minimize $\mathrm{CO}$ production, and allows a developer to eventually advise different ratio settings for the reference conditions. Tracing the U-curves, obviously, could be helpful not only in characterizing a fixed system but also to compare the performances of different burners in the development phase. Finally, since combustion behaviour is strongly system related, the impact of other components on combustion can also be spotted: the heat exchanger size and volumes, for example, would be interesting parameters to be evaluated in future appliance-oriented research.

\section{Conclusions}

The transition through a more environmentally friendly energy system will have an impact on grid gas quality. In fact, gaseous fuels composition is expected to significantly vary in the next years due to several factors, as the injection of biogases and hydrogen on the gas grid. This will help mitigating the problems related to renewable energy fluctuation and reducing the overall $\mathrm{CO}_{2}$ emission, but the impact of gas quality variation on each element of the production-to-consumption chain has to be evaluated. Premixed burners need particular attention in this context, because its design is very complex and currently based mainly on trial and error approach and empirical observations. Each gas device has its own characteristic response to varied inlet gas quality, so any future requirement for increased tolerance on gas composition and heating value will require a tailored activity to optimize the device design. Active combustion control systems could facilitate the transition process, but these systems are not completely mature to deal with all fuel components such as hydrogen. As consequence, a widening of the gas specification range would require an increased fuel flexibility to the gas burner itself. In this context, it is very useful to generate knowledge on the premixed burners behaviour under variable fuel quality, aiming to assist future appliance-oriented developments. Laboratory testing was conducted on a domestic premixed boiler system with the aim to gather more information on combustion behaviour at gradually varying air/gas mixture composition: a test protocol oriented to overcome the mere testing with limit gases was implemented. The tests were run at varying fuel/air ratio at fixed fuel composition, but can be taken as a basis for further testing on different fuels. The results allowed to detect a $\mathrm{CO}$ versus $\mathrm{CO}_{2}$ figure assuming a common macroscopic shape ("U-curve") for all reference conditions. The gas/air ratio is confirmed to play a fundamental role on the combustion quality, thus fuel gas composition variations shifting this ratio can significantly increase the $\mathrm{CO}$ production. The tested system resulted to be more fuel flexible at lower thermal loads: appliance developers should take care about fuel flexibility along whole modulation range, since the response can be strongly variable. The proposed U-curve tracing is a fundamental first step in characterizing the system fuel flexibility and detecting the preferable air/gas ratio for minimization of $\mathrm{CO}$ production. Furthermore, the method is applicable not only to the characterization of a fixed system but also to detection of the impact of different components on the combustion performance. In conclusion, the future burner design process will necessarily take into consideration an increased fuel quality range, and new test protocols and regulations need to be developed accordingly. 


\section{References}

[1] European Commission, EU Reference Scenario 2016 - Energy, transport and GHG emissions - Trends to 2050(2016).

[2] VHK for European Commision, Eco-design of Boilers - task 4 - Technical Analysis (2007).

[3] European Parliament, Directive 2009/125/EC (2009).

[4] R. Judd and D. Pinchbeck, Compendium of Hydrogen Energy, vol. 4, p.165-192 (2016).

[5] E. Hengevelda, . W. van Gemert, J. Bekkering and A. Broekhuis, Biomass Bioenerg, vol. 67, p. 363-371(2014).

[6] Department of Energy and Climate Change UK, Biomethane into the Gas Network: A Guide for Producers (2009).

[7] A. Singhal, Biomethane to Natural Gas Grid Injection: A Technological Innovation System Analysis, KTH Stockholm, MSc Thesis (2012).

[8] H.M. Altay, S.Park, D. Wu, D. Wee, A.M. Annaswamy and A.F. Ghoneim, Proc Combust Inst, vol. 32, p. 1359-1366(2009).

[9] Maarten Hoeijmakers, Viktor Kornilov, Ines Lopez Arteaga, Philip de Goey and Henk Nijmeijer, Proc Comb Inst, vol. 35, p.1073-1078(2015).

[10] P. Nitschke-Kowsky, Impact of hydrogen admixture on installed gas appliances, Kuala Lumpur 2012 World Gasconference (2012).

[11] SGC - Swedish Gas Technology Centre, Basic data on biogas (2012).

[12] Bord Gáis Networks, Report on Gas Quality arrangements in Republic of Ireland(2008).

[13] European legislation EN 437 - Test gases - Test pressures - Appliance categories.

[14] DNV GL commissioned by Ministry of Economic Affairs Netherlands, Requirements for gas quality and gas appliances (2015).

[15] Ernest Orlando Lawrence Berkeley National Lab, Natural gas variaility in California: environmental impacts and device performance (2009).

[16] UK Govenment, Future arrangements for Great Britain's gas quality specifications; Government Response (including Summary of Responses) to consultation on future arrangements for Great Britain's gas quality specifications, UK Government (2007).

[17] B. Slim, Should we add hydrogen to the natural gas grid to reduce $\mathrm{CO}_{2}$ emissions? (consequences for gas utilization equipment), 23rd World Gas Conference Amsterdam (2006).

[18] Health and Safety Laboratory - HSE UK, Injecting hydrogen into the gas network - a literature research (2015).

[19] José M. N. Rodrigues and Edgar C. Fernandes, Stability Analysis and Flow Characterization of Multi-Perforated Plate Premixed Burners, 17th International Symposium on Applications of Laser Techniques to Fluid Mechanics (2014).

[20] VHK for European Commision, Eco-design of Boilers - task 2 - Market Analysis(2007). 